\section{The Neo-Cartesian Revival: A Response}

\author{
Peter Harrison \\ Bond University
}

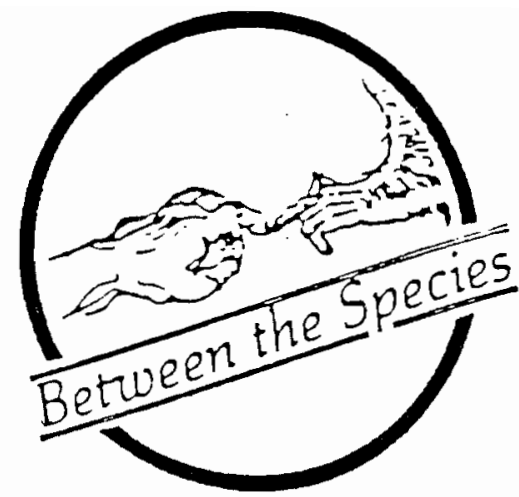

the practice of nailing research animals to boards for vivisection without benefit of anaesthesia." The implication is that from assumed facts about animalsthat they do not feel pain, for example-certain moral consequences directly follow. However, to arrive at conclusions about how animals ought to be treated, we must be armed with more than facts. 'Some moral theory must come into play. Indeed one's view of morality will determine which facts are morally relevant. For those who subscribe to some crude form of utilitarianism, according to which the morality of acts is judged by whether they conduce overall to physical pleasures or pains, putative facts about animal pain may well be the sole determinant of how animals ought to be treated. It might follow, for such utilitarians, that if animals do not feel pain, then there are no strictures on how they may be treated. However, for those who, like myself, subscribe to an alternative moral theory, animal pain is but one element among others. In my view there are moral considerations independent of the vexed question of animal pain which ought to govern our treatment of nonhumans. Thus, even if animals do not feel pain, it does not directly follow for those committed to some nonconsequentialist theory of ethics that we can treat animals how we wish. ${ }^{2}$

The fact that the question of animal pain and the moral status of animals are distinct issues can be further illustrated by reference to the treatment of animals in case in any detail, but given that it is not presented in its best light in the preceding article, readers may wish to consult it for themselves.

\section{The Moral Implications of Animal Pain}

Perhaps the most serious misconception in Professor Pluhar's paper is her contention that if Peter Carruthers and I have our way, "scientists might as well return to
(C) Peter Harrison, 1992

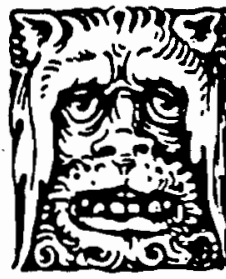

\section{PHILOSOPHY}


the seventeenth century. It is by no means clear, however frequently it may be asserted, that Descartes "had a profound effect on the practice of vivisection." The sources offered for this common view, if any are offered at all, are usually those contemporary French writers who describe the gruesome experiments carried out by the Cartesians at Port-Royal. ${ }^{3}$ However there are equally compelling counter-instances of Cartesians who preached kindness to animals. ${ }^{4}$ Moreover, for this argument to work, it would have to be shown that Cartesians pursued animal experimentation with more vigour and cruelty than did their non-Cartesian counterparts. To my knowledge, no one has established this. Indeed it would be surprising if they could, for in the seventeenth century it was simply not necessary to go to the extent of denying that animals felt pain in order to justify experimenting on them. Whether animals felt pain or not was irrelevant, given the almost universal belief that animals had been placed in the world for the service of mankind. It was this view, combined with the Baconian understanding of nature, which informed the practice of vivisection. Only with the advent of Bentham's utilitarianism did the purported capacity of animals to feel pain become morally significant. Again, it is clear that one's treatment of animals need not be determined solely, if at all, by whether they are capable of feeling pain.

\section{Mental States and the Relevance of Physical Similarities}

We now turn to my apparent covert commitment to Cartesian dualism. This is ascribed to me on the basis of examples put forward to show that pain is a mental state (a claim which, I confess, could have been stated more explicitly, but which should not be confused with another which Professor Pluhar erroneously attributes to me, that pain is "primarily psychological," whatever that means). There are few, if any, philosophers who would contest this assertion. What is at issue is the relation between such mentalistic statements as "I am in pain" and physicalistic statements of the kind " $X$ 's nervous system is presently in such and such a physical state." My position with respect to the relation of the mental and the physical is indeed dualist, but one may be a dualist without subscribing to Descartes' ontology of dual substances. Leibniz is a case in point, and some would argue that epiphenomalists are dualists of a kind. There are, in addition, a number of modern versions of dualism which differ from the classical formulation of Descartes. 5 "Dualists" in this context are simply those who consider propositions about mental states to differ from propositions about physical states in terms of both meaning and reference. Admittedly, dualism, Cartesian or otherwise, does have difficulties, but in my view these are less intractable than those of alternative theories of mind. In any case, arguments that animals are not subjects of such mental states as pain do not necessarily rely upon a dualist theory of mind. As Pluhar herself indicates, earlier this century it was actually opponents of dualism who, influenced by linguistic philosophy, challenged the meaningfulness of all mentalistic statements. In view of this confusion it might be worth sorting out some of the implications of various theories of mind, particularly with respect to the argument from physiological similarities.

It seems to me that the most a dualist can say is that under specific conditions, certain neurological structures seem necessary for mental events of certain kinds. ${ }^{6}$ (I mearr empirically necessary, not logically necessary.) To make the much more difficult case that the same neurological structures are sufficient for mental experiences, we need to subscribe to some theory of mind according to which, in principle, all propositions about mental events are logically deducible, or empirically discoverable, from propositions about physical states. ${ }^{7}$ A materialistic theory of mind, according to which mental states are nothing but physical states of the brain, would be most suitable. However, even if some form of physicalism is correct, and if, through some unimaginable scientific breakthrough, we are able to infer mental states from physical states, it may still turn out that the subjective experience of pain in one human individual may turn out to be represented by a different physical state from that which obtains in another human being experiencing pain. Thus no amount of knowledge of brain states would ever shed light on mental states. But further, even granting all of these "ifs," we would never be in a position to know what an animal's mental states were, because despite the most complete knowledge of their physical states, given that their nervous systems differ from ours in significant ways, and given that we have no independent access to their mental states (assuming they have them), we would have no way of constructing the hypothetical map which would allow us to read off mental state from physical state. Thus even the theory of mind which most favours the view that the similarity 
of animals' neural structures to our own is a relevant consideration in determining whether they feel pain, must be supplemented with additional assumptions.

When we turn to those psychologists whose research apparently involves "the induction of grief, anxiety, anguish and psychosis in nonhumans," the relevance of our discussion of alternative views of mind becomes apparent. It is clear that some materialistic theory of mind is often tacitly, and hence uncritically, accepted by the scientific fraternity. Symptomatic of its influence is an increasing tendency in psychology to convert mental criteria into physical criteria. Physical criteria can be measured and manipulated in a way that mental criteria cannot, and physical criteria yield "objective" knowledge. A classic case is the treatment of dreaming in psychological literature. The sole criterion for the occurrence of a dream is a waking report. However, some psychologists, attempting to establish links between measurable physical states and reports of dreams, have tended to displace the mental criterion in favour of some physical criterion, in this case, a period of rapid eye movement (REM). But as Norman Malcolm has ably demonstrated, however interesting the correlations between these physical events and subjects' reports of dreams, such connexions can only be established by continual reference to the original, mental criterion of dreaming. ${ }^{8}$ The illicit substitution of "period of REM sleep" for "dream" leads psychologists into a discussion of some phenomenon which only indirectly pertains to what we routinely refer to as "dreaming." The criterion of "dreaming" as we commonly use the term, is emphatically not some measurable physiological state, however convenient this latter characterisation might be for psychologists.

In animal psychology the tendency to make these kinds of illicit substitutions is endemic. Hence, as Pluhar has indicated, we find such labels as "grief," "anxiety," "anguish," and "psychosis" applied to animals. However, the criteria for the use of these terms are not, as in the case of human use, reports of mental state but, rather, particular physical syndromes which correspond roughly to those physical states of human beings who make reports of the psychological phenomena concerned. These subtle substitutions can only be meaningful if their reference is restricted to those physical characteristics which serve as criteria for their application or if the standard use of the terms as applied to humans is altered so that these terms refer not to mental states but to other physical criteria thought to correlate with those states. Either way, the use of such terms sheds no light on the mental lives of nonhumans and is at best highly equivocal, at worst, hopelessly muddled.

The whole body of "respectable evidence" to the effect that animals make "choices," incidentally, is predicated upon a similar linguistic confusion. ${ }^{9}$

In the light of this discussion, we can return to what prompted the charge of Cartesian dualism-my discussion of the psychology of pain. Reference was made to this literature to illustrate one simple principle: if two human individuals, who presumably have very similar neural structures, experience the same painful stimulus in different ways, the subjective experience of pain is simply not a direct function of neuroanatomy. (Aristotle, Spinoza and Ryle, incidentally, would hardly have supported my general position. Ryle, in particular, was a notorious anti-dualist. These philosophers were cited merely to show there is general agreement that pain is not a sensation.)

To conclude this discussion of the relevance of physical affinities, let me make it clear that I do not deny the significance of "the neurophysiological similarity of human and nonhuman species." This similarity, however, can admit of two quite distinct interpretations. I welcome Pluhar's announcement that we have $98.4 \%$ of genetic material in common with chimpanzees (although the statistic is somewhat misleading).${ }^{10}$ We can add to this the observation of Sir John Eccles that "in the [human] neocortex no special structural or physiological properties have been identified that distinguish sharply a human brain from the brain of an anthropoid ape." 11 What these similarities demonstrate is that the achievements of human consciousness are vastly disproportionate to the neurological differences which exist between ourselves and our nearest relatives. We do not characteristically observe our simian cousins producing works of art, writing literature, composing music, or building cities, which are, on average, $98.4 \%$ as good as ours. There is in the animal world nothing to compare with the products of the conscious mind, however much evolutionary biologists want to stress the continuities between human and animal species. (Chimpanzees' constructions of two- and three-term expressions in ASL do not, in my view, compare favourably with the works of Shakespeare. $)^{12}$ Nor need we be committed to any particular theory of mind to see the force of this disproportion. We may, like 
Descartes, postulate the existence of some spiritual substance which, allied to the human brain, accounts for the difference. Alternatively, we may want to argue that the unique products of human culture are direct functions of very small brain differences. But even if we adopt this second view, we are admitting that the relevant statistic is not $98.4 \%$ similarity, but the $1.6 \%$ difference. There simply is no proportionality between physical and mental worlds.

\section{Sensation and Consciousness}

In the final section of my paper I am alleged to have made a "stunning turn," conceding that nonhumans can experience pain. At least I am in illustrious company, for Descartes and I stand jointly charged with wanting to have it both ways, in that we are supposed to have asserted both that animals are not conscious and that they are capable of having sensations. The difficulty here is that words like "sensation" carry with them what Wittgenstein called "grammatical illusions"-in this case the belief that all sensation is conscious. (We have already witnessed this in the parallel instances of animal "anxiety," "grief," etc.) As Descartes himself was at pains to point out, albeit in vain, sensations need not be conscious. ${ }^{13}$ Animals, in his view, have sensations, but they are not aware of them. There is no ambiguity here, it is simply a matter of attending closely to how these words are used. (Leibniz's "apperception" was an attempt to avoid the misunderstanding engendered by the use of "sensation.") ${ }^{14}$ The point I am making, at any rate, is not, as Pluhar erroneously believes, that animals experience genuine pains and then forget them but, rather, that animals lack the self-conscious identity which is a prerequisite for conscious experience. The sentence which is cited in support of my stunning turn-'I am not implying here that painful experiences which are forgotten were never painful to start with"refers only to human experience, as should be clear from the original context.

Pluhar has placed a similarly unfortunate construction on my argument concerning learning in Protozoa. My observations about Protozoan habituation were to illustrate the principle that learning does not require consciousness, not that all learning was of the simple Protozoan kind. Rather than multiply examples beyond necessity, let me simply cite another author on this question:
On its own, the behavioural evidence is ambiguous, since persistence of a response after the stimulus has ended, memory, anticipation and learning are readily explained in terms of simple neural mechanisms which do not require the postulation of any form of subjective experience on the part of the animal. ${ }^{15}$

\section{Free Will and Determinism}

To holding "the undefended assumption that the capacity to choose is incompatible with the deterministic thesis," I plead guilty. I also concede that the classic problem faced by incompatibilists, like myself, is "how to construe an uncaused choice in a meaningful way." The classic counter is, of course, that free agents are the causes of their own actions. The problem of freedom and determinism, like the mind-body problem, is not one which I claim to have solved. All I have done is to opt for a philosophically-defensible thesis of human freedom which is consistent with my overall position. ${ }^{16}$

For the benefit of those who, with Professor Pluhar, believe that an action can be both determined and freely chosen, let me rephrase the argument which links pain and choice: If animal behaviour is externally caused (i.e., not caused by animals acting as agents), then we have some good reasons for thinking that animals do not suffer pain. Despite Pluhar's claim to the contrary, this is not an instance of attempting to prove a thesis by appealing to its premises. ${ }^{17}$ The only genuine ground for complaint is the "if" used in the formulation of this argument. If Pluhar can show that my view of free-will is wrong, then the argument fails. But while incompatibilism of the kind outlined above continues to be a serious philosophical option (although, as I conceded in the original paper, not an uncontroversial one), my case remains plausible.

\section{The Relevance of Evolutionary Theory}

Professor Pluhar is quite justified in chastising me for having given a rather simple account of natural selection. Indeed, my own case assumes the more sophisticated version which she presents. Yes, it is true that not every trait of every species confers some kind of selective advantage. Accordingly, when Pluhar states that it is "simply false to say that debilitating pain, depression, anxiety, etc., do not threaten our survival," 
I am in full agreement with her. My point is that the human species has something which compensates for this-namely, a culture which insulates us from selection pressures which might otherwise lead to our demise. Culture enables us to "carry" these, and perhaps other, negative traits. It seems less likely that animals, who do not enjoy the benefit of this cultural buffer, would be able to perpetuate similar disadvantageous traits.

It must also be pointed out that while evolutionary theory might in principle allow for the fact that some traits confer no selective advantage, it is not clear, in practice, when evolutionary biologists would be justified in abandoning the search for such explanations, for a researcher could never be in a position to distinguish between a trait which conferred an as yet unknown selective advantage, and one which was simply not advantageous. We can see the strength of this if we consider the example provided by Pluharthe behaviour of a cat or dog which ceases to eat on the death of a companion. It might reasonably be argued that one half of a superannuated breeding pair is no longer in a position to enhance the survival of the species. On the contrary, having fulfilled its reproductive function, it is now competing for food resources with other pairs which have reproductive potential. Not eating in this situation turns out to be advantageous to the species. Now, it does not really matter whether this account works or not. The point is that such behaviour is not intrinsically "mysterious" to those who deny that animals have subjective states analogous to human grief, because there will always be the possibility of some such overlooked explanation.

Professor Pluhar might respond at this juncture with the cry of "false dilemma." This "grieving" behaviour, she might point out, is both adaptive and indicative of mental grief. Rather than trump the false dilemma with Occam's razor, let me just point out that to introduce mentalistic explanations is immediately to move beyond the bounds of evolutionary theory, for it is behaviours, not mental states, which adapt, and it is only physical entities which can be the subjects of natural selection.

\section{Conclusion}

To conclude, I wish to offer some brief comments on Pluhar's final verdict on my argument. In her judgement, it consists of "a series of inadequately supported statements, hasty generalisations, and question-begging assumptions." I'm not sure about the hasty generalisations, but the remarks about the "inadequately supported statements" and the "questionbegging assumptions" are revealing. On the first head, there were, indeed, a number of inadequately supported statements in my piece. This does not mean that they are insupportable. My views on the nature of free will, for example, were not supported, but the running in this department has been made by others. The same applies to my assumptions about the nature of mind. The important thing in these cases is that arguments are available. Moreover, in the construction of a plausible argument-which is all I have claimed for my case-it is perfectly legitimate to make assumptions, provided that they are not clearly false. A critic must show that such assumptions are erroneous, not merely contentious. While Professor Pluhar has competently rehearsed some standard objections to a number of my assumptions, she has not shown any of them to be false.

On the second head, Pluhar seems to confuse the demonstration of the consistency of a position with begging the question. My argument functions in part by showing that if we take a certain position with respect to (say) free will, and add to this a commitment to a particular philosophy of mind, we may be led to certain remarkable conclusions about animal consciousness. These, in turn, may mesh neatly with a certain type of theodicy. The fact that these positions are mutually supportive actually strengthens the argument. It follows that an effective criticism would have to demonstrate that (a) my premises are false, or (b) the implications I have drawn from those premises are false, or (c) the various philosophical positions which serve as my premises are inconsistent with each other. Professor Pluhar has failed on each of these counts.

\section{Notes}

\footnotetext{
${ }^{1}$ This not least because, as Hume famously pointed out, no "ought" is deducible from an "is."

${ }^{2}$ Philosophers as divergent as Aquinas and Kant denied the relevance of animal pain, while insisting at the same time that we ought to refrain from cruelty to animals. More recently, some writers on animal welfare have avoided references to pain in the search for more objective criteria to inform the treatment of animals. See, e.g., D.M. Broom, "Indicators of Poor Welfare," British Veterinary Journal 142 (1986) $524 \mathrm{f}$. For a brief statement of the kinds of moral considerations which I consider to be relevant to our treatment of nonhuman animals, see Peter Harrison, "Do Animals Feel Pain?," Philosophy 66 (1991) 25-40, part v; cf. Patrick Bateson,
} 
"Assessment of Pain in Animals," Animal Behaviour 42 (1991) 827-39, p. 836.

The utilitarian approach, in any case, suffers from other embarrassments. While there is doubt about animals' subjective experiences of pain, there must be doubt about how they ought to be treated. Moreover, utilitarianism leads to a conflict of positive duties tow ard animals: Do we save the bird from the cat? For a systematic account of the limitations of a utilitarian approach to the issue, and of some of the implications of a contractualist ethics, see Peter Carruthers, The Animals Issue: Moral Theory in Practice (Cambridge: Cambridge University Press, 1992).

${ }^{3}$ The standard sources are Joseph Lavalée, Letters of a Marmeluke (London, 1804), p. 106; N. Trublet, Memoirs pour servir d' histoire de la vie et des ouvrages de $M$. de Fontenelle (Amsterdam: Mrac-Michel Rey, 1761), p. 115. Nicholas Fontaine also wrote of Cartesians who justified vivisection by claiming that animals did not feel pain: see $\mathbf{L}$. Rosenfield, From Beast-Machine to Man-Machine, (New York: Octagon, 1968), p. 54.

${ }^{4}$ See, e.g., John Norris, Essay Towards the Theory of the Ideal or Intelligible World (London: Pr. for S. Manship, 1704), pp. 59,100. Also Louis Racine, cited in Rosenfield, BeastMachine to Man-Machine, p. 53.

${ }^{5}$ For some modern versions of dualism, see The Case For Dualism, ed. J. Smythies and J. Beloff (Charlottesville: University Press of Virginia, 1989).

${ }^{6}$ Even this may be doubtful. We would generally say, for example, that a striate cortex is necessary for conscious visual experiences. However, some human individuals seem to be able to see without one. See David Paterson, "Is Your Brain Really Necessary?,"World Medicine 3, May, 1980, 21-4. The argument that certain neural structures are at least sufficient for certain conscious experiences would, in any case, only apply to human mental states. The brains of birds, for example, also lack a striate cortex, despite behaviour which suggests that they have visual experiences. I should add that some dualists believe in the possibility of disembodied existence. Under these conditions, obviously no physical state would be a necessary condition of a mental state.

${ }^{7}$ Epiphenomenalists would also accept this, but in my view the position of epiphenomenalism is neutral with respect to arguments about animals' consciousness.

${ }^{8}$ See Norman Malcolm, Dreaming (London: Routledge and Kegan Paul, 1959), ch. 13.

${ }^{9}$ I should point out that I do not deny the heuristic value of these locutions. For many reasons, both practical and scientific, it might be helpful to speak of animals as if they were subjects of mental states. But we ought not confuse the heuristic value of such expressions with their truth. Of course, some might wish to argue that, for example, measures of "stress" (qua physical phenomenon) should inform our treatment of animals. This is fine as far as it goes, but such arguments could not be justified by the further claim that "stress" is an index of pain.

${ }^{10}$ A significant amount of our DNA seems to play no active role in genetic specification.

11 John Eccles, The Human Mystery (Berlin: Springer, 1979), p. 234.

${ }^{12}$ It is not entirely clear to me why Pluhar has taken my ironic reference to chimpanzees and ASL as some kind of shameful admission. I can only assume that she has misunderstood the language criterion for consciousness. At this point, the only claim I will make for language use is that it is a dramatic example of the discontinuity between humans and other animals.

${ }^{13}$ See Peter Harrison, "Descartes on Animals," The Philosophical Quarterly 42 (1992) 219-27. Peter Carruthers makes the same point with his notion of "nonconscious experience."

${ }^{14}$ Leibniz, incidentally, for a time a least, shared Descartes' views about animal consciousness. Discourse on Metaphysics, XXXIV, tr. George Montgomery (Chicago: Open Court, 1902); cf. Correspondence with Arnauld, in Ibid., pp. 156, 220.

${ }^{15}$ Patrick Bateson, “Assessment of Pain in Animals," 834.

${ }^{16}$ The standard defence of self-determinism was provided by Thomas Reid, Essays on the Active Powers of Man (New York: Garland, 1977). For a more recent discussion see Agency and Necessity, ed. A. Flew and G. Vesey (Oxford: Blackwell, 1987).

${ }^{17}$ Pluhar makes this complaint because she confuses the logical relations which obtain between three distinct propositions: (1) X is a machine, (2) X cannot feel pain, (3) The behaviour of $X$ is externally determined. On these relations see John Cottingham, "A Brute to the Brutes?': Descartes' Treatment of Animals," Philosophy 53 (1971) 551-61.

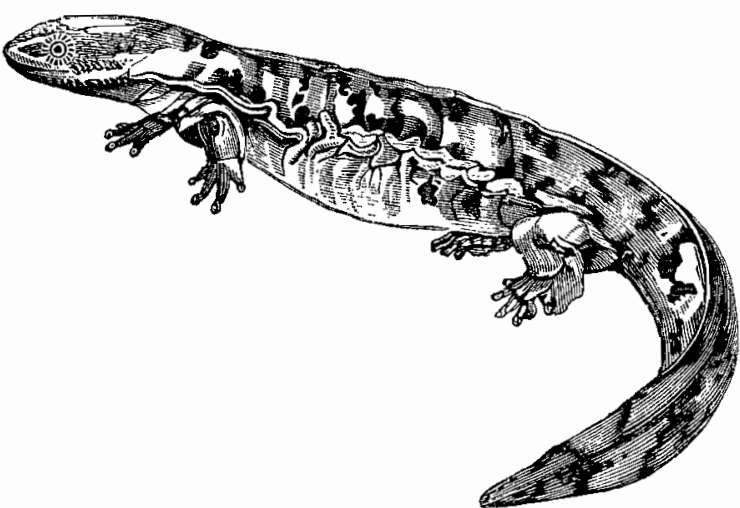

